\title{
The Dynamical Environment About Asteroid 25143 Itokawa: Target of the Hayabusa Mission
}

\author{
D.J. Scheeres*S. Broschart† S.J. Ostro and L.A. Benner ${ }^{\ddagger}$
}

\begin{abstract}
This paper explores the dynamical environment about Asteroid 25143 Itokawa, using the shape and rotation state model recently estimated from radar imaging data by Ostro et al. (2004). In 2005 the Japanese spacecraft Hayabusa will reach Itokawa and begin a period of vicinity operations about that body. The existence of this model can be used to prepare for the Hayabusa spacecraft rendezvous with that asteroid, enabling a more rapid transition from arrival to the start of intensive science and sampling operations. The Hayabusa mission also yields a unique opportunity to validate the shape inversion procedures from radar data described in Hudson (1993). We find that there are stable orbiting options for the Hayabusa spacecraft about Itokawa.
\end{abstract}

\section{The Hayabusa Mission and Itokawa}

In 2005 the Japanese Space Agency's (JAXA) Hayabusa spacecraft will rendezvous with Asteroid 25143 Itokawa. In its subsequent mission it will descend to the asteroid surface several times to acquire samples, and return them back to the Earth. Motivated by this rendezvous, a radar-based shape model and rotation state has been estimated for Itokawa by Ostro et al. (2004). The existence of this model allows the dynamical environment about this asteroid to be explored prior to rendezvous, potentially enabling a more rapid transition from arrival at the asteroid to the start of intensive science and sampling operations. The Hayabusa mission also yields a unique opportunity to validate the shape inversion procedures from radar data described in Hudson (1993).

This paper will first discuss the general geometric properties of the Itokawa shape model and its constant density gravity field. Following this, a detailed discussion of the dynamical environment about this body will be given, using the Hayabusa spacecraft as a motivational example. In particular, we will compute the surface environment, the dynamics environment close to the body, the dynamics environment far from the body, incorporating solar radiation pressure, and the predicted orbit stability of a Hayabusa-like spacecraft. We find that it is feasible for the Hayabusa spacecraft to orbit this asteroid, assuming a density of $2.5 \mathrm{~g} / \mathrm{cm}^{3}$ and some specific mass and area values for the spacecraft. Stable orbit geometries, however, are limited to lie in the sun terminator plane.

In June 2004, additional observations of Itokawa were made by Ostro and Benner. While not completely processed as of yet, they do indicate additional surface structure on the asteroid not seen (due to viewing geometry) during the previous observations. When these new observations are finalized, the analysis contained in this paper will be revised and should be available in advance of the 2005 rendezvous.

\section{Computed properties from the shape}

Given the detailed polyhedral shape of Itokawa we can compute a variety of quantities associated with that body. For all our discussions we assume a body-fixed coordinate frame with origin at the body center of mass and aligned with the principal axes of inertia of the asteroid (all computed assuming a uniform density throughout). We use the usual convention that the $x$ axis is along the smallest moment of inertia (longest axis), the $z$ axis is along the largest moment of inertia (shortest axis), and that the $y$ axis lies along

\footnotetext{
*Associate Professor, The University of Michigan, Department of Aerospace Engineering, Ann Arbor, MI $48109-2140$ USA, 1-734-615-3282 (tel), 1-734-763-0578 (fax), scheeres@umich.edu

$\dagger \mathrm{PhD}$. Candidate, The University of Michigan

$\ddagger$ Jet Propulsion Laboratory, California Institute of Technology
} 
the intermediate moment of inertia. Also, where applicable, we have assumed that the body rotates about its maximum moment of inertia (consistent with the fit to the radar data) with a rotation period of 12.132 hours.

The overall dimensions of the asteroid shape in each of the principal directions are:

$$
\begin{aligned}
& x:-0.2728 \rightarrow 0.2753 \\
& y:-0.1550 \rightarrow 0.1572 \\
& z:-0.1352 \rightarrow 0.1399
\end{aligned}
$$

with overall lengths of $0.5481,0.3122$, and $0.2751 \mathrm{~km}$ in the $x, y$, and $z$ directions, respectively (see Fig. 1 for the shape of Itokawa). Note, these overall lengths measure the maximum difference between coordinate values for the shape. The total surface area of the asteroid is $0.437 \mathrm{~km}^{2}$, and the total volume of the asteroid is $2.41 \times 10^{-2} \mathrm{~km}^{3}$. Associated with the volume is the mean radius of the body, $r_{M}=0.1791 \mathrm{~km}$, defined as the radius of a sphere with equivalent volume.

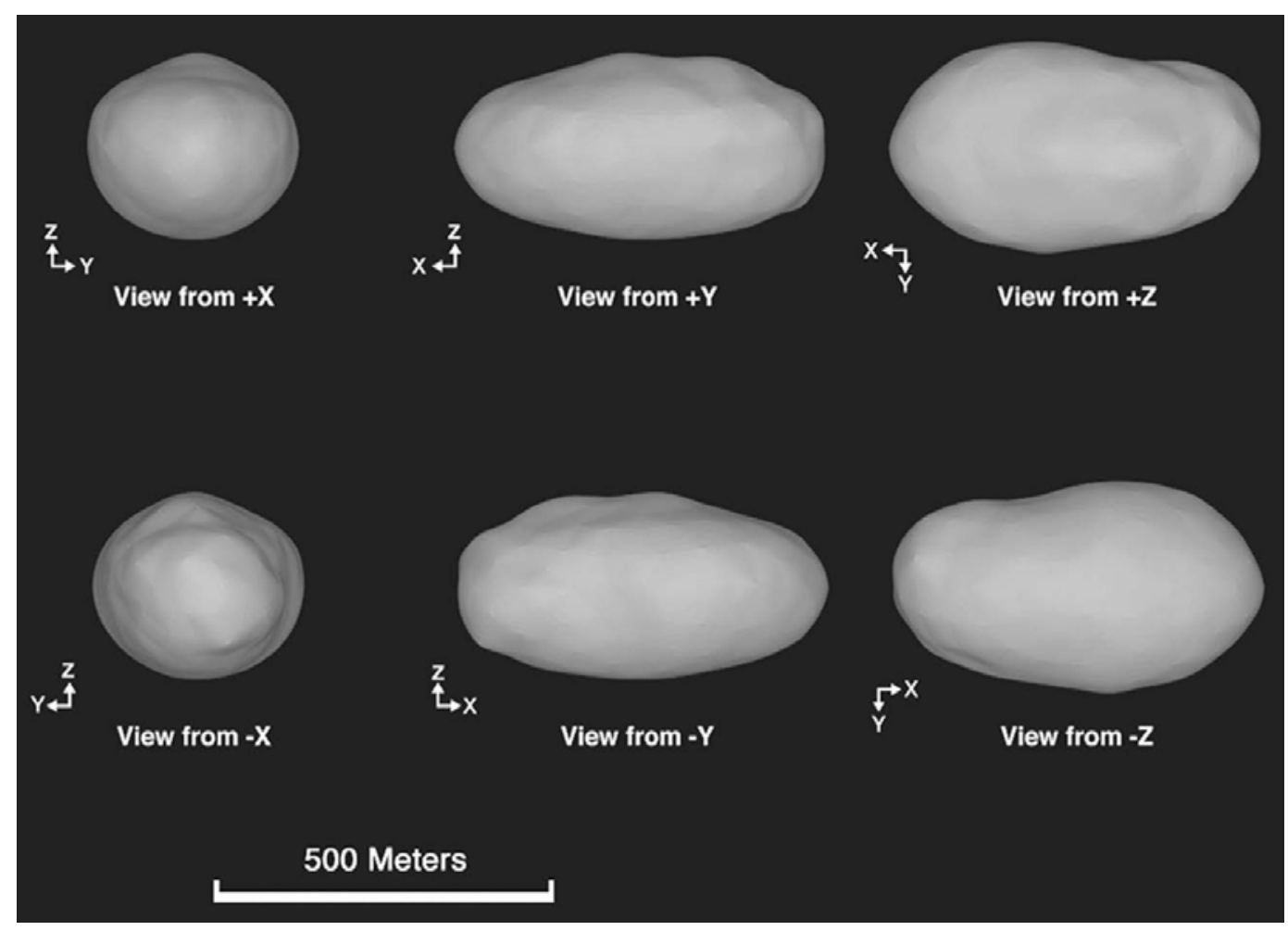

Figure 1. Shape of the Itokawa model (Ostro et al. 2004)

The higher-order mass distributions of the body, which include the inertia moments and the gravitational coefficients up to an arbitrarily high order, can be computed using the methodology outlined in Werner (1997). We find the moments of inertia:

$$
\begin{aligned}
& I_{x} / M=8.29 \times 10^{-3} \mathrm{~km}^{2} \\
& I_{y} / M=1.948 \times 10^{-2} \mathrm{~km}^{2} \\
& I_{z} / M=2.050 \times 10^{-2} \mathrm{~km}^{2}
\end{aligned}
$$

where we only quote the moments of inertia divided by the total mass of the body, which is currently unknown. From the moments of inertia we can solve for the ellipsoid with the same moments of inertia, and we find a body with semi-major axes of $0.2815 \times 0.1526 \times 0.1348 \mathrm{~km}$, which are close to the overall dimensions of the body.

Directly related to moments of inertia are the second degree and order gravity coefficients. These are directly computed to be:

$$
C_{20} R_{s}^{2}=-6.6133 \times 10^{-3} \mathrm{~km}^{2}
$$




$$
C_{22} R_{s}^{2}=2.7972 \times 10^{-3} \mathrm{~km}^{2}
$$

where $R_{s}$ is the arbitrary normalization radius. A gravity field expanded to degree and order 4 is given in the Appendix; a higher order gravity field can be obtained from the author on request. These gravity coefficients are close to but distinct from, the values expected if the body had an ellipsoidal shape defined by the above semi-major axes. A measure of the asteroid's shape and gravity field is defined in Hu and Scheeres (2004) as:

$$
\sigma=\frac{I_{y}-I_{x}}{I_{z}-I_{x}}
$$

For Itokawa this value is $\sigma=0.9165$. A body with $\sigma=1$ has a prolate inertia matrix while one with $\sigma=0$ has an oblate matrix, thus we see that Itokawa is very close to having a prolate shape index.

The computations given above are all exact for the body, under the uniform density assumption. In light of results from the NEAR mission (Yeomans et al. 2000), this assumption seems reasonable. In order to discuss the dynamical space about the body, we must add an additional assumption about the density of the asteroid. This can only be determined by measuring the total mass of the body, but meanwhile the radar returns from Itokawa place a constraint on the surface density of material (Ostro et al. 2004). Based on these returns, we assume a density of $2.5 \mathrm{~g} / \mathrm{cm}^{3}$ in the following, realizing that all the subsequent computations will have to be revised once the actual density of Itokawa is measured.

\section{Derived dynamical properties for surface, close proximity, and vicinity motions}

Under the density assumption a number of dynamical constraints for natural and spacecraft motion about Itokawa can be placed on the surface environment, the close proximity dynamical environment, and the environment in the vicinity of Itokawa, where solar perturbations become relevant. Assuming a uniform density for Itokawa of $2.5 \mathrm{~g} / \mathrm{cm}^{3}$ its gravitational parameter equals $\mu=4.01 \times 10^{-9} \mathrm{~km}^{3} / \mathrm{s}^{2}$. This value varies proportionally with density.

\section{A. Surface Environment}

Following the methodology outlined in Scheeres et al. (1996), we compute the apparent slope over the surface of the asteroid, the total gravitational plus centripetal accelerations over the body, and the necessary and sufficient speeds for ejecta to escape from the asteroid.

Itokawa has a very relaxed surface, with slopes ranging up to a maximum of $27.0^{\circ}$, less than the traditional "angle of repose" for a granular material. The average slope over the body is $9.1^{\circ}$ and $95 \%$ of the body has slopes less than $18^{\circ}$. Fig. 2 shows the slope distribution over Itokawa.

The maximum total surface acceleration (combining gravitational and rotational accelerations) is $1.236 \times$ $10^{-4} \mathrm{~m} / \mathrm{s}^{2}$ and the minimum is $0.996 \times 10^{-4} \mathrm{~m} / \mathrm{s}^{2}$, for a variation of $24 \%$ from minimum to maximum. These accelerations can also be broken into components normal to the local surface and tangential to the local surface. These distinctions are important when electrostatic forces and the sliding motion of particles are considered. The accelerations normal to the surface range from $0.923 \times 10^{-4} \rightarrow 1.236 \times 10^{-4} \mathrm{~m} / \mathrm{s}^{2}$. The accelerations tangent to the surface range from $\sim 0 \rightarrow 0.471 \times 10^{-4} \mathrm{~m} / \mathrm{s}^{2}$.

The surface environment can also be described by defining the necessary and sufficient surface launch speeds for escape from the asteroid (neglecting solar perturbation effects). The necessary launch speeds are the absolute minimum speed a particle must have if it is to escape from the asteroid, i.e., any launch speed less than this ensures that the particle will not leave the asteroid and will reimpact at some point in the future. These necessary speeds range from $0.127 \rightarrow 0.165 \mathrm{~m} / \mathrm{s}$ over Itokawa's surface. The sufficient launch speeds, on the other hand, are speeds that ensure that a particle launched with this speed in a direction normal to the surface will escape from the asteroid. These speeds range from $0.171 \rightarrow 0.246 \mathrm{~m} / \mathrm{s}$ over the surface. At each point of the asteroid, values between these speeds define launch speeds where the final evolution is not known. The differences between these speeds range from $0.036 \rightarrow 0.082 \mathrm{~m} / \mathrm{s}$. To be appropriately conservative, a surface rover on Itokawa should limit its jump speeds to less than the minimum "necessary" speed, in this case to less than $0.127 \mathrm{~m} / \mathrm{s}$, which would guarantee that a rover will not escape from the asteroid. 


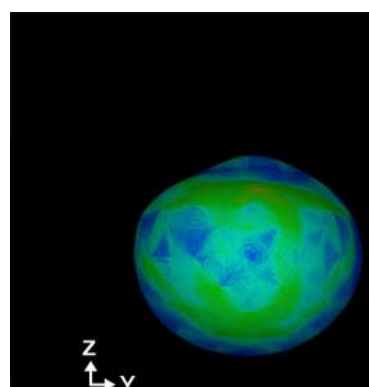

View from $+\mathbf{X}$

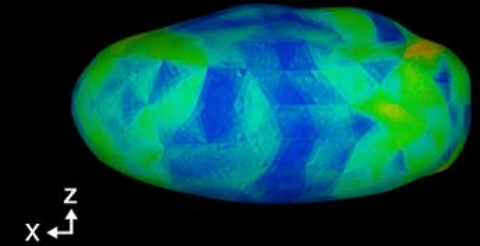

View from $+Y$

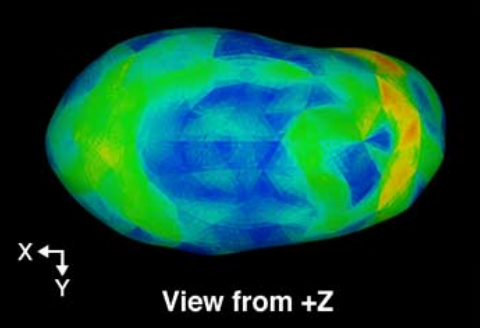

$z$

$\mathrm{Y}^{\mathrm{A}}$

View from - $\mathbf{X}$

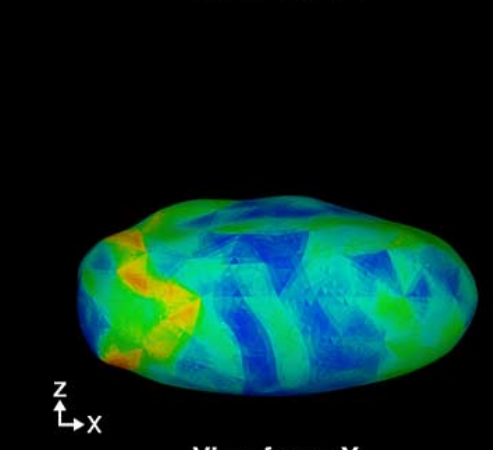

View from -Y

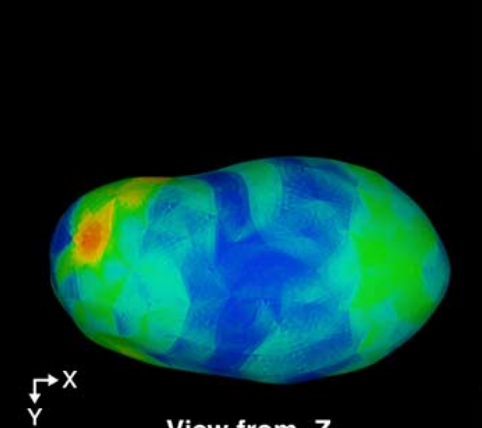

View from -Z

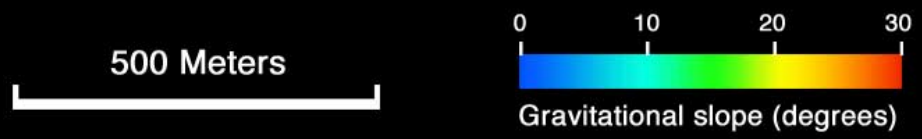

Figure 2. Slope values mapped onto the surface of the Itokawa model (Ostro et al. 2004)

The global stability of surface motion can also be characterized using the method described in Guibout and Scheeres (2003). To strictly apply this method, we must model Itokawa as an ellipsoid, an approximation that is rather good in this case. Using the ellipsoid parameters defined earlier we can show that at Itokawa, due to its slow rotation rate, loose material should preferrentially accumulate at its poles. Thus, it may be preferrable to sample regolith from the polar regions of Itokawa, barring other operational complications.

\section{B. Gravitational Dynamical Environment}

First we evaluate the dynamical environment close to Itokawa neglecting the solar radiation pressure and solar gravity perturbations. This neglect is not valid for the Hayabusa spacecraft, for which the effect of solar radiation pressure must be accounted for, even for motion close to the body. Thus, the results in this section only apply to larger natural particles with size greater than $\sim 10$ centimeters, such as ejecta blocks which may be lofted into orbit about Itokawa following an impact.

The simplest parameterization of the close proximity dynamical environment can be made by computing the "resonance radius" of the body, which we define as the distance at which the point mass gravitational attraction of the body equals the centripetal acceleration due to the rotation of the asteroid. This is computed as $r_{\text {res }}=\left(\mu / \omega^{2}\right)^{1 / 3}$, where $\omega$ is the rotation rate of Itokawa, equal to $1.4386 \times 10^{-4} \mathrm{rad} / \mathrm{s}$. For Itokawa this radius is $r_{\text {res }}=0.5787 \mathrm{~km}$.

A more precise measure of the close proximity environment can be found by computing the orbits synchronous with the rotating body (Scheeres 1994, Scheeres et al. 1996). For a near-ellipsoidal body such as Itokawa there will only be four such points, corresponding to true circular orbits about Itokawa with orbit period exactly equal to the Itokawa rotation period. These orbits appear as equilibrium points in the equations of motion about the asteroid when stated in the body-fixed (i.e., rotating) frame. Thus, their 
coordinates can be given in the body-fixed coordinate frame $(x, y, z)$ :

$$
\begin{array}{ll}
E_{1}: & 0.5987,-0.0046,-0.00006 \mathrm{~km} \\
E_{2}: & -0.0120,0.5704,0.0002 \mathrm{~km} \\
E_{3}: & -0.5997,-0.0100,-0.0002 \mathrm{~km} \\
E_{4}: & -0.0156,-0.5699,0.0002 \mathrm{~km}
\end{array}
$$

Figure 3 shows the location of these points in the Itokawa-fixed frame. The stability of these equilibrium points has been computed and we find that all four are unstable, making this a "Type II" asteroid (applying the criterion from Scheeres 1994). The points $E_{1}$ and $E_{3}$ have a simple hyperbolic unstable manifold with characteristic times (i.e., time for an initial error to grow by a factor of 2.7) of 3.69 and 3.50 hours, respectively. There are also two oscillation frequencies about each of these equilibrium points, with periods of $\sim 11.5$ hours. The points $E_{2}$ and $E_{4}$ are complex unstable with characteristic times of 20.9 and 14.9 hours, respectively, with an associated period of $\sim 17$ hours for the spiral motion. These points also have an additional oscillation mode (in the direction parallel to the rotation pole) with a period of 12 hours. The presence of four unstable orbits indicates that motion in close proximity to Itokawa will be subject to destabilizing influences from the gravity field, the exception being retrograde orbits with high inclinations (on the order of $135^{\circ} \rightarrow 180^{\circ}$ ), which is expected from the theory of asteroid orbiters (Scheeres et al. 2000).

In $\mathrm{Hu}$ and Scheeres (2004) a non-dimensional parameter that serves as a combined measure of the effect of asteroid gravity and rotation rate is defined, $\left(I_{z}-I_{x}\right) \sigma / r_{r e s}^{2}$, with a value of $\sim 0.033$ for Itokawa. Using the results from $\mathrm{Hu}$ and Scheeres (2004), we estimate that near-circular, direct orbits will be stable out to a distance of $\sim 2^{2 / 3} r_{\text {res }}$ from the center of Itokawa, which corresponds to an orbit radius of $0.92 \mathrm{~km}$ (see Fig. 3). We note that this is an approximate limit only and that motion outside of this radius may still be subject to destabilizing interactions through higher-order resonances.

\section{Gravitational plus Solar Dynamical Environment}

Next we discuss the dynamical environment further from the asteroid, in the realm where solar gravity and radiation pressure begin to dominate the motion of ejecta and spacecraft. We can split this discussion into two parts, the effect of solar gravity on motion about the asteroid and the combined effect of solar gravity and radiation pressure on motion. We note that for motion of larger ejecta, it is sufficient to only consider solar gravity, however for the motion of small ejecta or a spacecraft solar radiation pressure dominates over solar tide.

The effect of solar gravity can most easily be delimited in terms of the Hill sphere about the asteroid. Since Itokawa is in an elliptic orbit about the sun (perihelion radius of $0.953 \mathrm{AU}$ and aphelion radius of 1.69 $\mathrm{AU})$, the size of the Hill sphere will fluctuate and can be computed to be:

$$
R_{H i l l}=d\left(\frac{\mu}{3 \mu_{S}}\right)^{1 / 3}
$$

where $\mu$ is the asteroid gravitational parameter, $\mu_{S}$ is the gravitational parameter of the sun, and $d$ is the distance between the asteroid and sun. For Itokawa we find that the Hill radius will vary between $30.7 \mathrm{~km}$ at perihelion and $54.5 \mathrm{~km}$ at aphelion. Using the analysis from Scheeres and Marzari (2002) we estimate that the semi-major axis of orbits which remain bound to the asteroid for at least several particle orbits range from $10.2 \rightarrow 18.2 \mathrm{~km}$ (the limits corresponding to perihelion and aphelion). These limits are well outside the limiting radius for strong interactions with the asteroid gravity field, but are only valid for a body insensitive to solar radiation pressure.

To include the effect of solar radiation pressure, it is necessary to specify the mass to projected area ratio of the object orbiting about the asteroid. The relevant parameter that describes the relative strength of the solar radiation pressure force is defined in Scheeres and Marzari (2002) as $\tilde{\beta}=3.84 / B / \mu^{1 / 3}$, where $B$ is the mass to area ratio of the orbiting body in $\mathrm{kg} / \mathrm{m}^{2}$, and $\mu$ is the gravitational parameter of the asteroid. When $\tilde{\beta} \ll 1$ the solar radiation pressure effect is small, but when it is equal to or greater than 1 its effect will be quite strong. For a spherical body with the same assumed density as Itokawa, an object with radius $0.7 \mathrm{~m}$ will have a $\tilde{\beta}=1$ and a mass to area ratio $B=2419 \mathrm{~kg} / \mathrm{m}^{2}$.

Based on pre-launch estimates, the mass to projected area ratio of the Hayabusa spacecraft is expected to be $\sim 33 \mathrm{~kg} / \mathrm{m}^{2}$. This leads to a solar radiation pressure parameter $\tilde{\beta} \sim 73$, indicating that this force will 


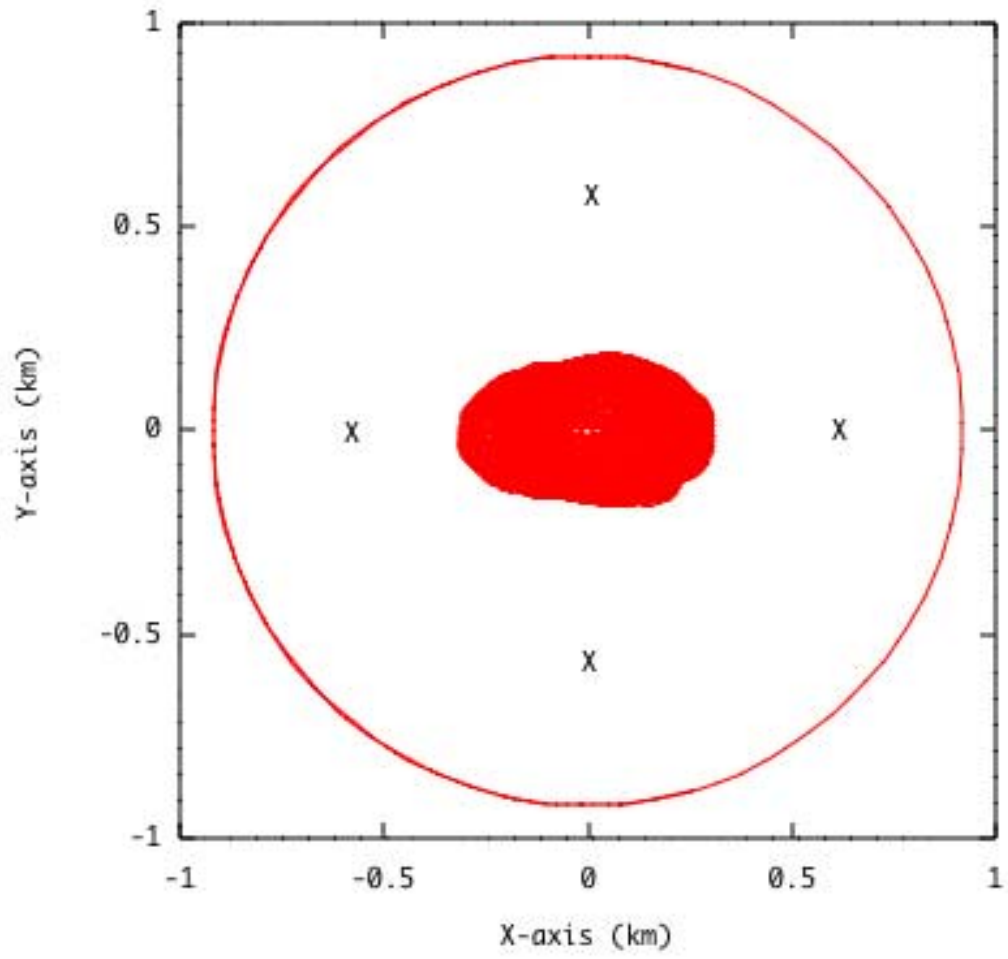

Figure 3. Pole-on view of Itokawa with locations of the equilibrium points marked with an ' $\mathrm{X}$ ' and the approximate stability limit for direct orbits marked with a circle.

be very relevant. As described in Scheeres and Marzari (2002), the Hill region is no longer spherical but is highly skewed away from the asteroid on the sun-side and towards the asteroid on the anti-sun side. For the Hayabusa spacecraft the sun-side libration points range between $1078 \rightarrow 1912 \mathrm{~km}$, while the anti-sunside libration points range between $5.2 \rightarrow 9.2 \mathrm{~km}$. We note the large disparity between these limits. Due to solar radiation pressure, the limiting semi-major axis for a spacecraft to be bound to the asteroid over several orbits is also considerably smaller, and ranges from $1.3 \rightarrow 2.3 \mathrm{~km}$. All the limits in the above ranges correspond to perihelion and aphelion.

We can also use these limits to predict the smallest sized regolith grains on the surface of Itokawa. As is currently suspected, small particles on asteroid surfaces will be subject to levitation forces due to the plasma environment on their surface (Lee 1996, Robinson et al. 2001). When such levitation occurs on a small asteroid, the particles can be placed into orbit and may potentially escape if the solar radiation pressure perturbation is large enough. If we assume a small levitated particle is a sphere with radius $r_{o}$, then its mass to area ratio will be approximately $B \sim 4 / 3 \rho r_{o}$. Applying the analysis in Scheeres and Marzari (2002) to this problem, we see that particles with radius less than $0.2 \mathrm{~mm}$ should escape from the body. Even if levitation is not an important effect, impacts on the asteroid would similarly loft small particles off the surface and subject them to the same perturbations. It will be interesting to see if the minimum grain size from the Hayabusa sample agrees with this calculation.

\section{Stability of spacecraft trajectories}

This analysis also predicts that the Hayabusa spacecraft's trajectory will be strongly perturbed by the solar radiation pressure. It is important to note that the Hayabusa mission will be using a "hovering" approach to their vicinity operations (Kubota et al. 2001, Broschart and Scheeres 2004), a decision that is very fortuitous 
for that mission. Despite this, we will still explore the dynamics of Hayabusa about Itokawa, in case a shift in operations plans is needed, or in case added scientific benefit can be gained by such a period of natural orbit dynamics.

First we note some particular facts concerning orbital motion in the presence of strong solar radiation pressure perturbation. All motion that occurs in the asteroid orbital plane will have a strong tendency for eccentricity to approach unity due to solar radiation pressure, while the semi-major axis remains constant (Scheeres 1999). Thus, such orbits tend towards impact trajectories. In general, as the spacecraft orbit inclination relative to the asteroid orbital plane increases, an evolving orbit will reach a maximum eccentricity less than unity. Still, these orbits tend to be unsafe, as any increase in eccentricity allows the trajectory to interact more strongly with the rotating Itokawa gravity field. This eccentricity oscillation effect diminshes until the orbit inclination relative to the asteroid orbit plane is $90^{\circ}$, with the orbit oriented so that it lies in the sun-terminator plane. This configuration results in a stable sun-synchronous orbit, and variations in the orbit eccentricity can be minimized. The most stable configuration for these orbits is designed as follows. The eccentricity of the orbit is chosen according to the rule:

$$
\begin{aligned}
e & =\frac{1}{\sqrt{1+\Lambda^{2}}} \\
\Lambda & =462 \frac{\sqrt{a}}{B}
\end{aligned}
$$

where the parameter $\Lambda$ is evaluated for Itokawa using the general formula given in Scheeres (1999). For the Hayabusa spacecraft, its value is $14 \sqrt{a}$, where $a$ is the semi-major axis in km. Thus, for an orbit with semi-major axis of $1 \mathrm{~km}$, an eccentricity of 0.07 is prescribed. For this eccentricity to minimize the variations also requires that the periapsis be oriented directly (90 degrees) below the orbit plane if the orbit angular momentum points away from the sun, and directly above the orbit plane if this orbit angular momentum points towards the sun. It is in general sufficient to just start the spacecraft trajectory in the sun terminator plane with a zero eccentricity and the resulting trajectory will have oscillations in eccentricity on the order of 0.14 for a $1 \mathrm{~km}$ orbit.

It is important to note that our numbers give a formal prediction of a "stability" interval about the asteroid for the Hayabusa spacecraft, between the capture radius for solar radiation pressure and the lower bound on stable, near-circular motion. Also, we have predicted the existence of stable retrograde orbits about this mass distribution. These results should be treated with caution, however, as there are non-trivial interactions between the solar radiation pressure and gravitational field perturbations of Itokawa.

To properly consider the interactions between the solar effects and the Itokawa gravity field we need to specify the asteroid's orbit in a frame oriented along its rotation pole. This is the natural frame in which to discuss orbit dynamics, as close proximity motion to the body is fundamentally organized by its rotation pole, and the preferred sun-terminator planes for motion more distant from the body need to be understood in this same reference frame.

\begin{tabular}{l||c|c}
\hline Element & Earth Ecliptic & Asteroid Pole \\
\hline Pole Lat. & $-84^{\circ}$ & $90^{\circ}$ \\
Pole Long. & $355^{\circ}$ & $\mathrm{N} / \mathrm{A}$ \\
Inclination & $1.7^{\circ}$ & $172^{\circ}$ \\
Asc. Node & $70.9^{\circ}$ & $0^{\circ}$ \\
Arg. Periapsis & $161^{\circ}$ & $330^{\circ}$ \\
\hline
\end{tabular}

Table 1. Elements in the Earth Ecliptic frame and the asteroid rotation pole frame

Thus, sun-terminator orbits at Itokawa will have an inclination in the Itokawa-based frame of $90^{\circ} \pm 8^{\circ}$. This is fortuitous, as a larger or smaller inclination can cause the orbit plane to precess independent of the sun-synchronous motion of the asteroid about the sun due to the effect of the asteroid oblateness, which can cause orbital instability. Indeed, we find that sun-terminator orbits of a Hayabusa spacecraft model at Itokawa remain stable and sun-synchronous for larger semi-major axes up to orbit radii of $2 \mathrm{~km}$ at perihelion (Fig. 4). We find that such orbits are the only feasible ones for motion about Itokawa. At lower semimajor axes, we find that the asteroid oblateness orbit precession effect becomes strong enough to force the trajectory to rotate out of the terminator plane, which in turn causes the orbit to become unstable (Fig. 5). 
For the Hayabusa spacecraft we find that retrograde orbits, which are extremely stable against gravitational perturbations, become destabilized due to the solar radiation pressure. This occurs as retrograde orbits lie close to the orbital plane, and hence the solar radiation pressure effect forces them to a unity eccentricity. This effect can be partly mitigated, however, for close as the gravity field will cause their argument of periapsis to have a large precession rate (Scheeres 1994), which will limit the maximum eccentricity which retrograde orbits reach. The combined effect of these perturbations causes the eccentricity to go through large variations, but not as large as if the central body were a simple sphere. We see this explicitly in Fig. 6 , where the first two trajectories are for the Hayabusa spacecraft incorporating solar radiation pressure, while the last figure shows the trajectories with no solar radiation pressure.

\section{Conclusions}

A study of the dynamical environment about Asteroid 25143 Itokawa, the target of the Japanese space mission Hayabusa, is given. This study is enabled by the existence of a radar-derived shape model of the body. We find that spacecraft motion about this body is highly unstable in general, although there are a class of orbits which can be safely flown in. For our model of the Hayabusa spacecraft and the asteroid, these are circular orbits at a radius of approximately $1 \mathrm{~km}$ in the sun-terminator plane. Orbits larger than this are subject to being stripped out of orbit by solar radiation pressure while orbits less than this are susceptible to destabilizing perturbations from the gravity field. New radar observations of Itokawa were recently taken, and indicate a non-trivial update to the shape model. A new version of this paper will be produced once these measurements are fully processed and a new shape model produced.

\section{Acknowledgements}

DJS acknowledges the support of NASA's Planetary Geology and Geophysics program through a grant with the University of Michigan. We thank Shigeru Suzuki and Eric DeJong, of the Jet Propulsion Laboratory, for the creation of Fig. 2.

\section{Appendix}

Following are the Itokawa gravity coefficients through order 4 (shown in Table I). These coefficients are normalized, as defined in Kaula (2000), computed with respect to a normalizing radius $R_{s}=0.302064 \mathrm{~km}$. The general form of the gravitational field can be expressed as:

$$
\begin{aligned}
U= & \sum_{l=0}^{\infty} \sum_{m=0}^{l}\left(\frac{R_{s}}{r}\right)^{l+1} P_{l m}(\sin \phi) \\
& {\left[C_{l m} \cos m \lambda+S l m \sin m \lambda\right], }
\end{aligned}
$$

where $P_{l m}$ are the normalized Legendre polynomials, $\phi$ is the particle latitude, and $\lambda$ is the particle longitude in the body-fixed frame.

\section{Bibliography}

Broschart, S.B. and D.J. Scheeres. 2003. "Control of hovering spacecraft near small bodies: Application to Asteroid 1998 Itokawa," Journal of Guidance, Control, and Dynamics, in press.

Hu, W. and D.J. Scheeres. 2004. "Numerical Determination of Stability Regions for Orbital Motion in Uniformly Rotating Second Degree and Order Gravity Fields," Planetary and Space Science 52: 685-692.

Hudson S. (1993) Three-dimensional reconstruction of asteroids from radar observations. Remote Sens. Rev. 8, 195-203.

Kaula, W.M. 2000. Theory of Satellite Geodesy, Dover Publishing.

Kubota, T., Hashimoto, T., Uo, M., Maruya, M., and Baba, K., "Maneuver Strategy for Station Keeping and Global Mapping around an Asteroid," Space- flight Mechanics 2001: Advances in the Astronautical Sciences, Vol. 108, 2001, pp. 769-779, AAS Paper 01-156.

Ostro, S.J., L.A.M. Benner, M.C. Nolan, C. Magri, J.D. Giorgini, D.J. Scheeres, S.B. Broschart, M. 


\begin{tabular}{cc|cc} 
Order & Degree & $C$ Coefficient & $S$ Coefficient \\
$l$ & $m$ & $C_{l m}$ & $S_{l m}$ \\
\hline 0 & 0 & 1.0 & - \\
1 & 0 & 0.0 & - \\
1 & 1 & 0.0 & 0.0 \\
2 & 0 & -.0922215 & - \\
2 & 1 & 0.0 & 0.0 \\
2 & 2 & .1351127 & 0.0 \\
3 & 0 & -.0001768 & - \\
3 & 1 & .0002468 & .0019196 \\
3 & 2 & -.0019275 & -.0047926 \\
3 & 3 & -.0122294 & -.0062563 \\
4 & 0 & .0397246 & - \\
4 & 1 & -.0018445 & -.0001744 \\
4 & 2 & -.0543996 & -.0023396 \\
4 & 3 & .0006763 & .0013784 \\
4 & 4 & .0589245 & .0058470
\end{tabular}

Table 2. Itokawa normalized gravity field coefficients through degree and order 4 for a constant density gravity field. A gravity field through degree and order 16 is available from the authors. Definition of the normalization constants can be found in Kaula (2000).

Kaasalainen, D. Vokrouhlicky, S.R. Chesley, J.-L. Margot, R.F. Jurgens, R. Rose, D.K. Yeomans, S. Suzuki, and E.M. De Jong. 2004. "Radar Observations of Asteroid 25143 Itokawa (1998 SF36)," Meteoritics and Planetary Science 39(3): 407-424.

Scheeres, D.J. 1994. "Dynamics About Uniformly Rotating Tri-Axial Ellipsoids. Applications to Asteroids," Icarus 110:225-38.

Scheeres, D.J., S.J. Ostro, R.S. Hudson, and R.A. Werner. 1996. "Orbits Close to Asteroid 4769 Castalia," Icarus 121:67-87.

Scheeres, D.J. 1999. "Satellite Dynamics about small bodies: Averaged Solar Radiation Pressure Effects," Journal of the Astronautical Sciences 47:25-46.

Scheeres, D.J., B.G.Williams, and J.K. Miller. 2000. "Evaluation of the Dynamic Environment of an Asteroid: Applications to 433 Eros," Journal of Guidance, Control and Dynamics 23:466-475.

Scheeres, D.J. and F. Marzari. 2002. "Spacecraft dynamics in the vicinity of a comet," Journal of the Astronautical Sciences 50(1): 35-52.

Werner, R.A., 1997. Spherical harmonic coefficients for the potential of a constant-density polyhedron. Comput. \& Geosci. 23, 1071.

Yeomans, D.K., P.G. Antreasian, J.-P. Barriot, S.R. Chesley, D.W. Dunham, R.W. Farquhar, J.D. Giorgini, C.L. Helfrich, A.S. Konopliv, J.V.McAdams, J.K.Miller,W.M. Owen Jr., D.J. Scheeres, P.C. Thomas, J. Veverka, and B.G. Williams. 2000. "Radio Science Results During the NEARShoemaker Spacecraft Rendezvous with Eros," Science 289:2085-2088. 

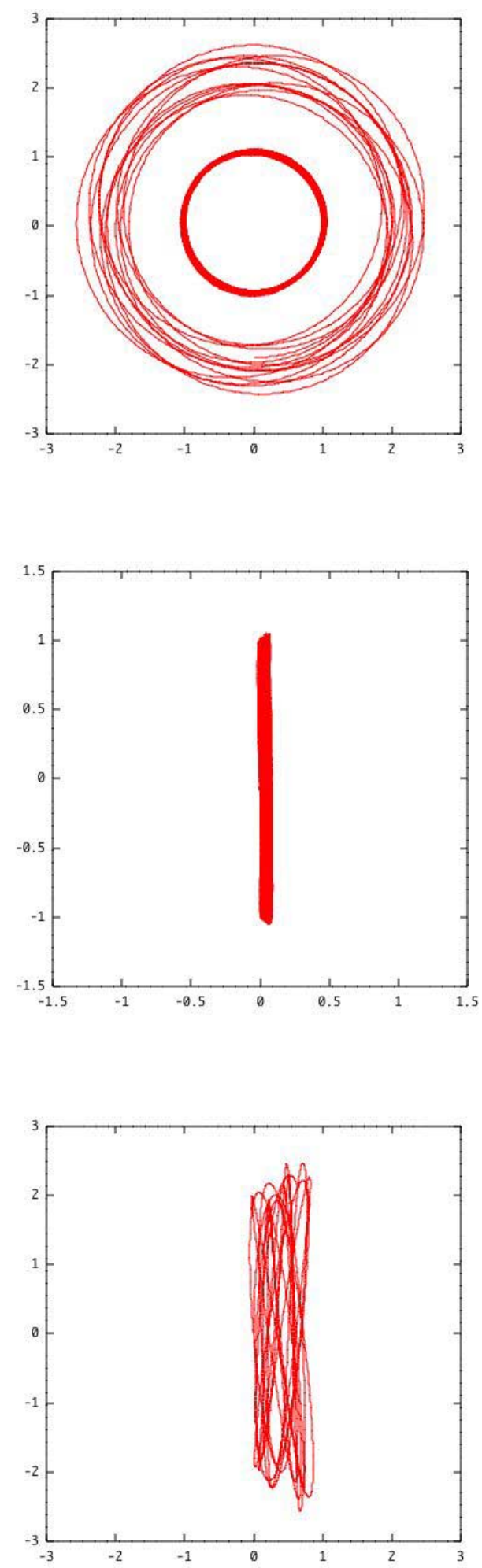

Figure 4. Integrated orbits of Hayabusa about Itokawa in the sun-terminator plane. Top: Initially frozen orbits with semi-major axis 1 and $2 \mathrm{~km}$ as viewed from the sun. Middle: Trajectory viewed from above the orbit plane, sun off along the negative horizontal axis, $1 \mathrm{~km}$ semi-major axis. Bottom: Same as Middle, but for $2 \mathrm{~km}$ semi-major axis. 

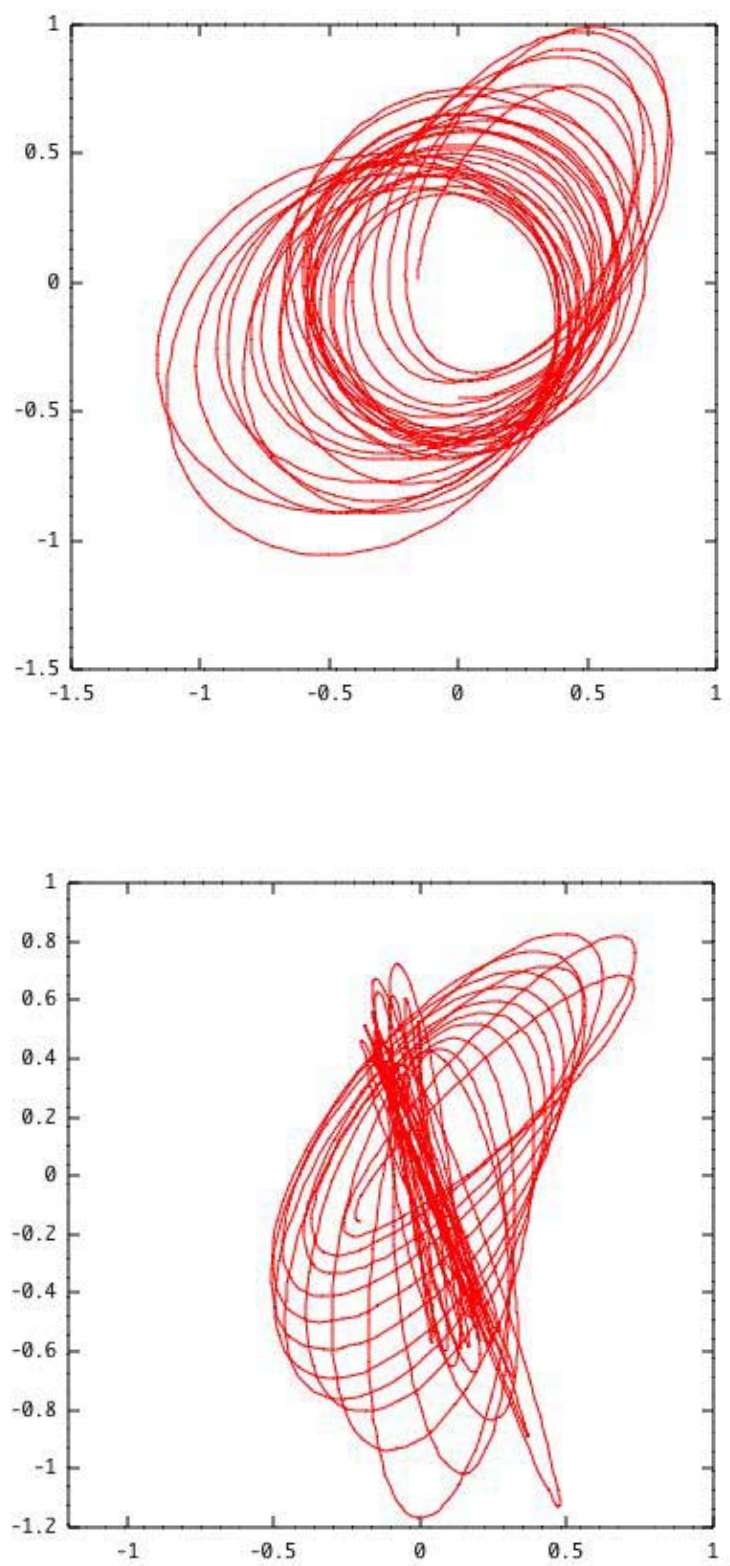

Figure 5. Integrated orbits of Hayabusa about Itokawa in the sun-terminator plane. Initially frozen orbit with semi-major axis $0.5 \mathrm{~km}$. Top: Trajectory viewed from the sun. Bottom: Trajectory viewed from above the orbit plane, sun is off along the negative horizontal axis. 

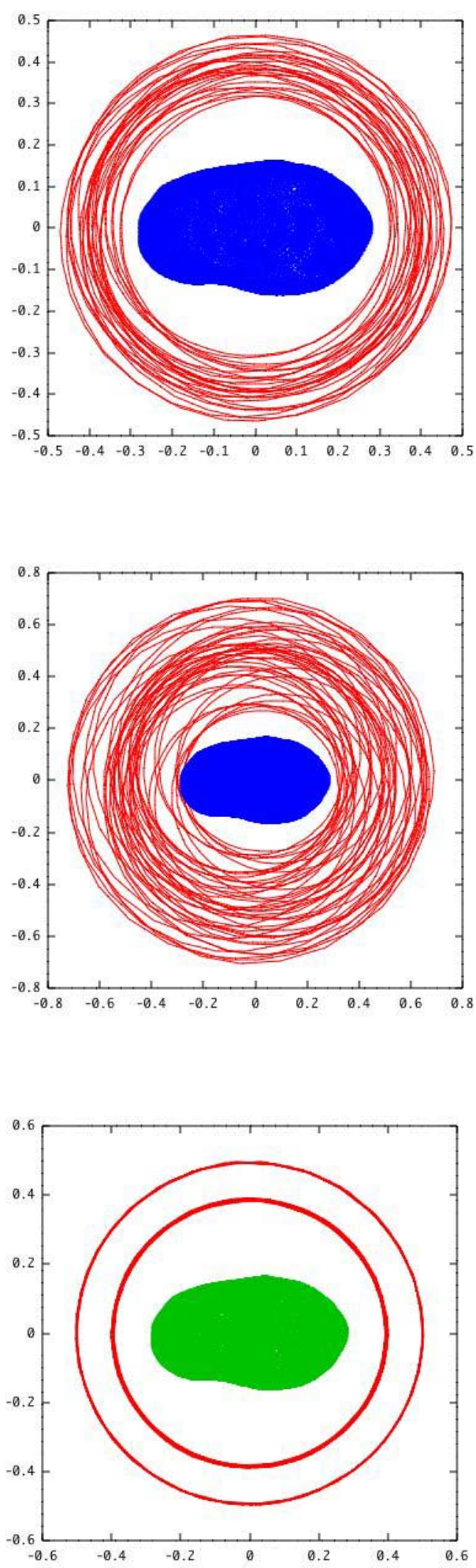

Figure 6. Integrated retrograde orbits of Hayabusa about Itokawa with and without solar radiation pressure. Top: Initially circular orbit with radius $0.4 \mathrm{~km}$. Middle: Initially circular orbit with radius $0.5 \mathrm{~km}$. Bottom: Initially circular orbits with radius 0.4 and $0.5 \mathrm{~km}$ and no solar radiation pressure. 